DISCUSSION/SIGNIFICANCE OF IMPACT: Intervention mapping allows for behavior change theories to be incorporated into counseling sessions with patients and their social support networks. This approach translates qualitative data into an evidence-based intervention which will be piloted in a randomized controlled trial (RCT) to determine feasibility for a larger RCT.

\section{Ascertaining the Medical Needs of Galveston County}

Sharon Croisant ${ }^{1}$, Krista Bohn ${ }^{1}$, and John Prochaska ${ }^{1}$

${ }^{1}$ The University of Texas Medical Branch

OBJECTIVES/GOALS: Data were collected or abstracted from a wide variety of sources related to health and health care needs to determine the current health status of the Galveston community including:

- Demographics

- Social Determinants of Health

- Health Care Access and Insurance Status

- Poverty and Socio-Economic Indicators Impacting Health

- Health Behaviors

- Chronic Disease

- Communicable Disease

- Birth Outcomes

- Mortality

- Cancer

- Data on Services Provided at UTMB

- Data on Services Provided through the Galveston County Health District

- Data on Services Provided through the St. Vincent's House Clinics, student-led clinics operated at a local non-profit organization

- Previous Galveston County Community Health Needs Assessment

- Identifying Gaps in Services

- Prevention Quality Indicator Data

METHODS/STUDY POPULATION: In addition to collection and analysis of secondary data, we also interviewed key stakeholders to solicit their input and recommendations. We met with leadership from St. Vincent's House regarding current services provided, perceived issues and concerns, and needs for improvements. We met with leaders from UTMB's academic enterprise to discuss the operation of our current student-led clinics as well as ways in which clinical practice experiences might be expanded and included more formally in the student curricula should the clinical capacity of St. Vincent's House also be significantly expanded. This would increase the number of services that could be offered at St. Vincent's and greatly increase the capacity for enrolling patients without relying on faculty volunteers to staff the clinics. We also met with UTMB leaders in a position to provide insight to issues that bridge the UTMB practice arena and public health and with Community Health leaders from the Galveston County Health District and Teen Health Clinics. Information Services leadership and Institute for Translational Science informatics faculty and staff were instrumental in determining what data could be abstracted from the Electronic Medical Record (without patient identifiers) to determine the specific need for services at St. Vincent's. RESULTS/ ANTICIPATED RESULTS: The City of Galveston has a population just under 50,000. Since 2010, the proportion of elderly has increased, and the proportion of families with younger children has decreased. Poverty is high at $22.3 \%$ for all people, and especially high for children at $32.1 \%$.
Poverty disproportionately affects racial and ethnic minorities, with $36.5 \%$ of the Black population living below the poverty level, compared to $25.5 \%$ Hispanic, $30.5 \%$ Asian, and $14.7 \%$ White. Home ownership is decreasing, and median rent costs have sharply increased. The percentage without health insurance is considerable, driven by educational attainment, age, and race. In $2017,>40 \%$ of renters spent more than $35 \%$ of their income on housing. Upwards of 2,650 reported not having access to a vehicle for transportation. While residents of Galveston County as a whole are less impoverished, those that are impoverished share marked similarities. Lower educational attainment, in particular failure to complete high school or obtain a college degree, are correlated with race. Lower educational attainment then is highly predictive of poverty and low income. The income inequality ratio, i.e., the greater division between the top and bottom ends of the income spectrum in Galveston County is higher than in Texas or the nation and has increased every year but one since 2010. Issues of concern for Galveston County include obesity, Type II diabetes, and disability. These are exacerbated by built and social environment issues such as food insecurity, limited access to healthy foods, and food deserts in some neighborhoods. Pre-term birth rates are higher in Galveston than in the state or nation, and approximately $40 \%$ of women do not receive prenatal care until the $2^{\text {nd }}$ or $3^{\text {rd }}$ trimester or receive no prenatal care at all. $8.4 \%$ of births are low-birth weight. Marked disparities by race and ethnicity exist for each of these indicators. Age-adjusted death rates for all-cause mortality are higher in Galveston County than they are in Texas or the United States. Perhaps of most concern are the rates of death from septicemia, which are nearly triple that of the U.S. and nearly double that of the state, and cancer. Cancer incidence is not particularly remarkable, however, cancer age-adjusted mortality rates for many specific cancers well exceed state rates. DISCUSSION/SIGNIFICANCE OF IMPACT: With a clearer picture of the medical and other needs impacting health or health care access for our community, all stakeholders and experts can provide more detailed recommendations about prioritizing care and especially, preventive care-much of which could conceivably be provided in St. Vincent's House clinics. Opportunities exist for enhanced practice and education opportunities for UTMB students from all schools. Preventive Care and Population Health practices can be brought to bear in novel practice settings that could serve as models for provision of integrated services. Social and other services provided by non-profit organizations can be coordinated and streamlined. It is our hope that the considerable data presented herein will enable stakeholders to begin to prioritize issues and to make some evidence-based decisions about the next steps in this process. Throughout the interview and data collection process, all stakeholders have expressed both enthusiasm and hope at the prospect of re-visioning how they can contribute to a process that will improve how we as a community care for our most vulnerable members. CONFLICT OF INTEREST DESCRIPTION: The authors have no conflicts of interest to disclose.

4523

\section{Assessing Depression in Puerto Rican Hispanic Patients Hospitalized with Heart Failure}

Ariel Gonzalez-Cordero ${ }^{1}$

${ }^{1}$ University of Puerto Rico-Medical Sciences Campus

OBJECTIVES/GOALS: Heart failure and depression are important public health problems. Depression has been identified as an independent risk factor for increased cardiovascular morbidities. It is estimated that 1 out of every 5 patients living with heart failure suffer from depression. (Kop, W. J.) Studies have found that Hispanic 
patients have a higher rate of heart failure than non-Hispanic whites. Current guidelines recommend that proper screening tools must be used to identify and manage major depression disorder in HF patients, however many of these patients go unrecognized in the medical setting. The prevalence, management, and outcomes of depression among PuertoRican Hispanics living with heart failure is unknown. Objective: The purpose of this study is to evaluate the relationship between heart failure and depression in Hispanics with heart failure METHODS/STUDY POPULATION: To this end, we will perform a secondary analysis of data from the PR CardiovascularSurveillance Study (PRCSS). We will extract personal data from 4,461 medical records of patients admitted with heart failure (ICD-9 Codes 428) at 21 hospitals in Puerto Rico, during the years 2007, 2009 and 2011. For statistical methods, we will implement chi-square and t-tests at a significance level of 0.05 RESULTS/ANTICIPATED RESULTS: We expect to see older aged women with higher NYHA and pro-BNP levels to be associatedwith diagnosis of major depression disorder and worse in-hospital outcomes DISCUSSION/SIGNIFICANCE OF IMPACT: With this study, we would like to raise awareness about depression in patients with heart failure, and its role in improving patient outcomes. Moreover, we would like to determine if there are gender-specific health disparities among Puerto Rican Hispanics with heart failure

4200

Assessment of differential access to patient online portal (POP) by socioeconomic status (SES) and its impact on asthma care and research

Young J Juhn ${ }^{1}$, Chung-il Wi, Euijung Ryu, Sunghwan Sohn, Miguel Park, Joy Fladager Muth, Hee Yun Seol, Katherine King, and Hongfang Liu

${ }^{1}$ Mayo Clinic

OBJECTIVES/GOALS: Patient online portal (POP) allows patients to access electronic health records (EHRs) and have efficient communication with their clinicians. We assessed disparities in access to POP by families with different SES and its impact on asthma research which is little known in the literature. METHODS/ STUDY POPULATION: A randomized controlled trial testing the efficacy of an EHRs-based clinical decision support (CDS) system was conducted at a pediatric primary care setting of Mayo Clinic. Asthma Control Test (ACT) questionnaire was administered to parents every 3 months through phone or email for this study after consenting, and reminders were sent to unanswered subjects through the POP. SES was measured by HOUSES (in quartiles), a validated individual-level SES index based on housing features (the higher HOUSES, the higher SES).The association of HOUSES with availability of POP access and missing ACT score rate was assessed. RESULTS/ANTICIPATED RESULTS: The mean age of 184 participants was 9.0 years (57\% male) and parents of 152 (83\%) children had POP. Only $68 \%$ of children from lowest HOUSES (Q1) had access to POP (vs. 74\% (Q2), 88\% (Q3), and 92\% (Q4; highest SES); $\mathrm{p}=.02)$. ACT score was completed by $144(78 \%), 150$ (82\%), 171 (94\%), and $164(95 \%)$ at each intervention conducted every 3 months with a total of 61 (33\%) missing at least once. Overall, children whose parents had access to POP had a lower missing rate in ACT score at all interventions during the study; $16 \%$ (those with access to POP) vs. $47 \%$ (those without), $13 \%$ vs. $44 \%$, $3 \%$ vs. $16 \%$, and $1 \%$ vs. $23 \%$ for $1^{\text {st }}, 2^{\text {nd }}, 3^{\text {rd }}$, and $4^{\text {th }}$ intervention, respectively $(\mathrm{p}<.007$ for all). DISCUSSION/SIGNIFICANCE OF IMPACT: There are significant disparities in access to POP by
SES defined by HOUSES which impact availability of ACT score resulting in a systematic bias in asthma research and potentially widening disparities in asthma care. CONFLICT OF INTEREST DESCRIPTION: NA.

4537

\section{Association between Geographic Socioeconomic Disadvantage and Incidence of Total Hip Replacement Surgery}

Rafa Rahman ${ }^{1}$, Joseph K. Canner ${ }^{1}$, Elliot R. Haut, MD, $\mathrm{PhD}^{1}$, and Casey J. Humbyrd ${ }^{1}$

${ }^{1}$ Johns Hopkins University School of Medicine

OBJECTIVES/GOALS: Total hip replacement (THR) improves function for those with arthritis, but not all patients have equal access to this elective procedure. To better geographically target healthcare resources, we explored whether geographic socioeconomic disadvantage is associated with incidence of elective THR. METHODS/ STUDY POPULATION: We performed a cross-sectional analysis of data in the state of Maryland from 2013-2019. We categorized 5 -digit zipcodes into national quartiles of socioeconomic disadvantage using the Area Deprivation Index (ADI). For each zipcode, we calculated the THR incidence rate using Maryland Health Services Cost Review Commission (HSCRC) inpatient and outpatient data in those age 65 years and older. We included only elective THRs. We analyzed the association between a zipcode's disadvantage quartile and THR incidence rate using multivariate linear regression, correcting for differences across zipcodes in gender, race, and ethnicity distributions, and distance to the nearest hospital performing THRs. RESULTS/ANTICIPATED RESULTS: We analyzed 414 zipcodes with overall average THR rate of 370.8 per 100,000 persons $>65$ yo per year. Relative to zipcodes in the least socioeconomically disadvantaged quartile, those in the second-least disadvantaged had 82.2 fewer THRs per 100,000 persons $>65$ yo per year, those in the second-most disadvantaged had 144.2 fewer, and those in the most disadvantaged had 207.4 fewer (all p65yo per year, those in the second-most disadvantaged had 136.2 fewer, and those in the most disadvantaged had 182.9 fewer (all $\mathrm{p}<.05$ ). DISCUSSION/ SIGNIFICANCE OF IMPACT: More socioeconomically disadvantaged areas have significantly lower rates of elective THR, independent of differences in demographics and hospital proximity. These findings show how disparities can affect access and outcomes, and should inform targeting of community-level education and intervention.

4070

Association of Interpersonal Processes of Care and Health Outcomes in Patients with Type II Diabetes Hadley Reid ${ }^{1}$, Olivia M Lin ${ }^{1}$, Rebecca L Fabbro ${ }^{1}$, Kimberly S Johnson ${ }^{1}$, Laura P. Svetkey, MD ${ }^{1}$, and Bryan C Batch ${ }^{1}$

${ }^{1}$ Duke University

OBJECTIVES/GOALS: 1 . Understand the association between patient perceptions of care measured by the Interpersonal Processes of Care (IPC) Survey and glycemic control, appointment no-shows/cancellations and medication adherence in patients with type II diabetes. 2. Determine how these relationships differ by race for non-Hispanic White and Black patients. METHODS/STUDY POPULATION: This is a cross-sectional study of a random sample of 100 White and 100 Black Type II diabetic patients followed in 